\title{
Resistance training decreases 24-hour blood pressure in women with metabolic syndrome
}

\author{
Ramires Alsamir Tibana', Guilherme Borges Pereira², Jéssica Cardoso de Souza', Vitor Tajra', \\ Denis Cesar Leite Vieira', Carmen Silvia Grubert Campbell', Claudia Regina Cavaglieri ${ }^{3}$ and Jonato Prestes ${ }^{1 *}$
}

\begin{abstract}
Background: The purpose of this study was to verify the effects of eight weeks of resistance training (RT) on 24 hour blood pressure (BP) in patients with and without metabolic syndrome (MetS).

Methods: Seventeen women volunteered to participate in this study, 9 with MetS (37.0 \pm 8.7 yrs; body mass $77.3 \pm$ $9.7 \mathrm{~kg}$; body mass index $30.3 \pm 4.2 \mathrm{~kg} \cdot \mathrm{m}^{-2}$ ) and 8 without MetS ( $35.1 \pm 7.2 \mathrm{yrs}$; body mass $61.3 \pm 8.1 \mathrm{~kg}$; body mass index $24.2 \pm 2.5 \mathrm{~kg} \cdot \mathrm{m}^{-2}$ ). Individuals were subjected to eight weeks (3 times/week) of whole body RT comprised of one exercise for each main muscle group with three sets of 8-12 repetitions of each subject's maximal load. A rest interval of one minute was allowed between sets and exercises. Twenty-four hour BP was measured by ambulatory blood pressure monitoring.

Results: Mean and diastolic night-time BP decreased $(-3.9 \mathrm{mmHg}, p=0.04 ;-5.5 \mathrm{mmHg}, p=0.03$, respectively) after eight weeks of training in MetS patients. This decrease was observed at 11:00 pm, 02:00 am (only diastolic), 07:00 am, and 6:00 pm. There was no training effect on BP in women without MetS.

Conclusions: Considering the elevation of BP as a contributor to the pathogenesis of MetS, and also to the increase of cardiovascular risk, this study supports RT as a non-pharmacological therapy in the management of BP control for MetS.
\end{abstract}

Keywords: Resistance training, Metabolic syndrome, Blood pressure

\section{Background}

Cardiovascular disease has been identified as a leading cause of death in the United States with medical costs and productivity losses reaching approximately $\$ 450$ billion. Additionally, more than 2 million Americans have a heart attack or stroke each year, and more than 800,000 of them die every year as consequence of cardiovascular problems [1].

Metabolic syndrome (MetS) is characterized by the grouping of several cardiovascular risk factors such as central obesity, dyslipidemia, insulin resistance, elevated glucose plasma levels and elevated blood pressure (BP) [2]. Moreover, it has been shown that the relative risk of cardiovascular disease and death in individuals with MetS is 1.78 higher than in individuals without MetS

\footnotetext{
* Correspondence: jonatop@gmail.com

${ }^{1}$ Graduate Program on Physical Education and Health, Catholic University of Brasilia (UCB), Brasilia, Brazil

Full list of author information is available at the end of the article
}

[3]. The prevalence of MetS has increased in developing countries in recent decades, while alarming data revealed a 32.0\% prevalence of MetS in the central region of Brazil [4]. Thus, the decrease of atherogenic dyslipidemia, prothrombotic state and BP is important to reduce the risk for cardiovascular diseases. Therefore, this reinforces the importance of developing effective and affordable strategies such as exercise programs to prevent and treat cardiovascular risks [5].

Current recommendations for the treatment of MetS and the primary prevention of cardiovascular diseases encourage the use of therapeutic lifestyle changes such as increasing physical activity, reducing dietary intake, and achieving weight loss [6-8]. Regarding physical activity interventions, few studies have reported the effectiveness of exercise programs alone for multiple cardiovascular risk factors [8-10]. Smutok et al., [11] compared the effects of 20 weeks of resistance training (RT) to aerobic training, and observed that both interventions resulted in increased

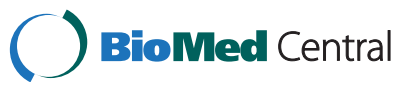


glucose tolerance and insulin sensitivity, with no changes in lipoprotein lipids and BP. Katzel et al., [12] found that aerobic exercise alone did not improve the lipid profile and BP. However, the HERITAGE Family Study revealed that 20 weeks of aerobic exercise training in patients with the MetS resulted in a decrease of $38 \%$ clinical BP [11].

To have clinical relevance, the decrease in BP should be maintained for many hours and sustained during daily activities [13]. Studies with RT revealed a significant post-exercise hypotensive effect, although most studies were limited to the analysis of BP during a specific period after exercise under laboratorial conditions, which is called clinical BP [14]. Thus, the use of ambulatory blood pressure (ABP) monitoring, which provides a spectrum of the $24 \mathrm{~h}$ hemodynamic load, has been recently expanded [15]. A recent meta-analysis suggests that moderate-intensity RT can reduce systolic blood pressure (SBP) and diastolic blood pressure (DBP), increase in $\mathrm{VO}_{2}$ peak, and decrease body fat and plasma triglycerides, both in normotensive and pre-hypertensive subjects [16]. However, the evidence on the chronic effects of dynamic RT on ABP monitoring remains understudied in patients with MetS.

Existing data regarding $24 \mathrm{~h} \mathrm{BP}$ and the post-exercise hypotensive effect are both scarce and controversial. Considering this, the aim of the present study was to verify the effects of eight weeks of RT on 24 h BP in patients with and without MetS. The initial hypothesis was that the RT program would be effective in decreasing BP during sleeping hours and daily activities in patients with MetS.

\section{Methods}

\section{Subjects}

Twenty-five middle-aged women volunteered to participate in the study. However, because of time constraints, eight subjects (three from the MetS group and five from the group without MetS) dropped out or did not complete the entire protocol. Therefore, nine patients with MetS and eight without MetS successfully completed the study. The inclusion criteria for participation in the study included age above 18 years, no RT experience, being free of clinical problems that could be aggravated by the protocol and ability to attend more than $85 \%$ of the training sessions. Exclusion criteria for the group without MetS included the chronic use of any medication and hypertension (SBP $>140 \mathrm{mmHg}$ and DBP > $90 \mathrm{mmHg}$ ). Additional exclusion criteria for women with and without MetS included regular exercise, subjects with physical disabilities, diagnosis of diabetes, cardiovascular diseases, musculoskeletal disease and recent smoking or drug/alcohol abuse. Resting 12-lead electrocardiogram recordings obtained from all subjects before participation in this study showed normal electrocardiographic patterns. Sedentary state was defined by the International physical activity questionnaire (IPAQ). The study protocol was approved by the Catholic University of Brasilia Ethics Committee (protocol \#376/2010), and patients gave written, informed consent.

Metabolic syndrome was defined according to the American Heart Association and National Heart, Lung, and Blood Institute scientific statement [17]. The criteria for diagnosis of the MetS was the presence of $\geq 3$ of the following characteristics: elevated waist circumference $(\geq 88 \mathrm{~cm})$, elevated triglycerides (TG) $(\geq 150 \mathrm{mg} / \mathrm{dl})$ or receiving drug treatment for elevated TG, reduced HDL cholesterol $(\leq 50 \mathrm{mg} / \mathrm{dl})$ or receiving medication for reduced HDL cholesterol, elevated BP $(\geq 130 \mathrm{~mm} \mathrm{Hg} \mathrm{SBP}$ or $\geq 85 \mathrm{~mm} \mathrm{Hg} \mathrm{DBP)}$ ) or receiving antihypertensive drug treatment with a history of hypertension, elevated fasting glucose $(\geq 100 \mathrm{mg} / \mathrm{dl})$ or receiving medication for elevated glucose. A modified $\mathrm{z}$ score was calculated for each variable using individual subject data using the Adult Treatment Panel (ATP) III criteria. The equations used to calculate the MetS $\mathrm{z}$ score were as follows: $\{\mathrm{z}$ score $=[(50-$ HDL $) / 11.8]+[($ TG -150$) / 66.2]+[($ fasting blood glucose - 110)/10.4] + [(waist circumference - 88)/ $9.2]+[($ mean arterial pressure -100$) / 8.7] / 100\}$.

\section{Study design}

The study investigated the effects of eight weeks of RT on 24-hour BP in women with and without MetS. The RT program was performed on three non-consecutive days of the week (Monday/Wednesday/Friday) and was comprised of three sets of 8-12 repetitions maximum (RM) of 12 exercises, and 1-minute rest intervals between sets and exercises performed. All testing and training sessions were conducted between 08:0009:00 pm. Before training and four days after the end of the RT all subjects underwent $24 \mathrm{~h}$ BP monitoring (Dyna-MAPA ${ }^{\circ}$, Cardios, Brazil). All subjects received instructions about the technique and position of the cuff during the $24 \mathrm{~h}$ BP monitoring. In addition, the subjects were encouraged to avoid smoking, alcohol, caffeine, unusual physical activity and to maintain their usual diet consumption (this was confirmed by a dietary recall follow-up). Individuals were asked to go to bed at 11:00 pm and awaken at 06:00 am on recording days.

\section{Biochemical parameters}

Participants reported to the laboratory between 08:0010:00 am, after an overnight fast, for blood withdrawal from the antecubital vein. Triglycerides, HDLcholesterol and glucose were measured by the following methods: enzymatic CHOP-POD, homogeneous HDLcholesterol and Hexokinase, respectively.

\section{Anthropometric and body composition evaluation}

Height and weight were measured for the calculation of the body mass index (BMI; body mass in $\mathrm{kg}$ divided by 
square of height in meters). All circumferences were obtained using a non-elastic tape; measurements were obtained in triplicate and then averaged. Neck circumference was obtained with the subject sitting with the head in the Frankfort horizontal plane position. A measuring tape was applied around the neck inferior to the laryngeal prominence and perpendicular to the long axis of the neck, while the minimal circumference was measured and recorded to the nearest $0.1 \mathrm{~cm}$ [18]. Waist circumference was measured at the midway level between the lower rib margin and the iliac crest [18]. The body fat percentage was determined by the Jackson and Pollock equation [19].

\section{Twenty-four hour blood pressure monitoring}

Twenty-four hour BP was measured in the non-dominant arm with an oscillometric monitor (Dyna-MAPA ${ }^{\circ}$, Cardios, Brazil) in accordance with manufacturer's instructions before the initiation of the training program and four days after the eight weeks of RT were finished. The monitor was programmed to perform measures every 15 minutes during daytime and every 30 minutes during the sleeping hours. All ambulatory measures of BP were performed during weekdays (i.e. Monday to Friday), and initiated between 9:00-10:00 pm. All participants were advised to maintain their habitual activities, refrain from programmed exercise for at least $72 \mathrm{~h}$ before the measures, and to stop any activity and relax the arm during each measurement. Data were calculated and analyzed as follows: mean of all measurements during the $24 \mathrm{~h}$ period, and mean of all measures performed during daytime and sleeping hours. The BP measurements were considered invalid for analysis if $>30 \%$ of the measurements were missing, if data were lacking for an interval of $>2 \mathrm{~h}$, or if the sleeping hours period was $<6$ or $>12 \mathrm{~h}$ [20]. Individuals were advised to control their liquid ingestion before data acquisition. Additionally, the cuff size was adapted to the circumference of the arm of each participant according to the manufacturer's recommendations.

\section{Resistance training program}

Before initiation of the experimental sessions, the volunteers completed two weeks of familiarization prior to testing [21]. During the familiarization weeks, individuals were advised regarding the execution of proper technique, and completed 3 sessions/week, with one exercise for each main muscle group (same exercises of the RT). Individuals performed 3 sets of 10-12 submaximal repetitions at $60 \%$ of estimated 10RM. Machines were from JOHNSON - USA.

The RT program consisted of 3 sessions/week for eight weeks. All training sessions were carefully supervised by three physical education professors (ratio of supervision 1:2 - 1 professor for 2 participants). Participants were required to complete at least $85 \%$ of the exercise sessions.
No major complications or cardiac events occurred during the study period. Figure 1 shows the exercise order that was strictly followed by both groups. The RT was divided into A (Monday) and B (Wednesday) and whole body (Friday) regiments. For both groups, abdominal crunches (three sets of 15 repetitions in all sessions) were included. For all listed exercises, three sets with 8-12 RM were performed [22], with a one-minute rest interval between each set and exercise. The average duration to complete one repetition was 3-4 s (both concentric and eccentric phases of the movement). The total exercise time was $\approx 40$ $50 \mathrm{~min}$ for all regimens. The number of repetitions and the loads used for each exercise session were recorded. The loads were updated when necessary to keep the number of repetitions within the same range of RM and to provide a progressive overload. Additionally, the participants were instructed to maintain their normal food intake during the research and correct breathing patterns were instructed to avoid Valsalva maneuver.

\section{Statistical analyses}

The normal distribution of the data was checked using the Shapiro-Wilk normality test and the Mauchly homoscedasticity test. The pre-intervention variables of the two groups were compared by using unpaired Student's $t$-test. The effects of the RT on the experimental groups were assessed by a dependent Student's $t$-test. Considering a power $(1-\beta)$ of 0.80 and an alpha error of 0.05 , the sample size used in this research allowed identifying a moderate effect size. $\mathrm{P}<0.05$ was accepted as significant, and data are reported as means \pm SD. Data were analyzed using the Statistical Package for Social Sciences (SPSS, v.19, Chicago, IL).

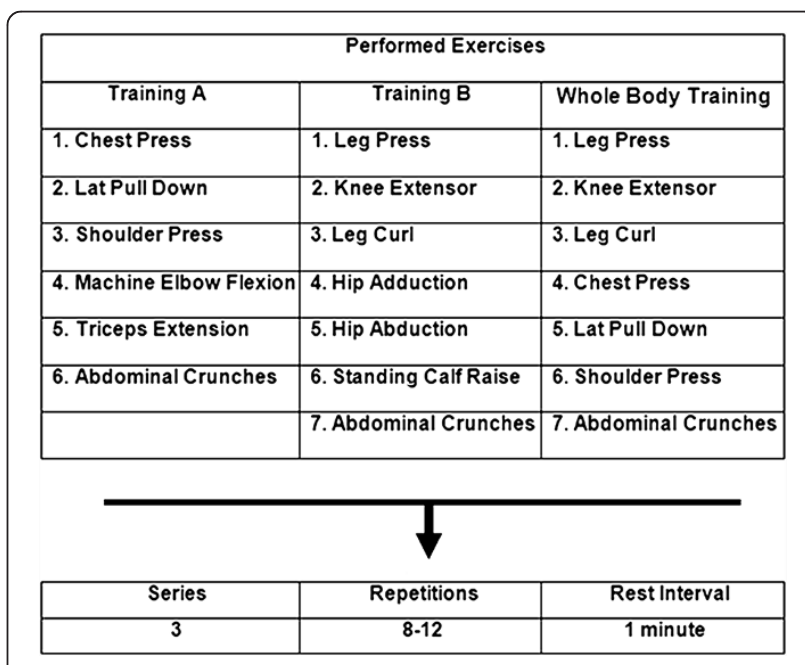

Figure 1 Division of the RT sessions throughout the study. For all exercises, 3 sets with $8-12$ repetitions maximum were performed with a 1-minute rest interval between each set and exercise. 


\section{Results}

General subjects characteristics are presented in Table 1. The MetS group presented higher values for body mass, lean body mass, fat body mass, body mass index, percentage body fat, neck circumference, waist and hip circumference and triglycerides as compared with the group without MetS.

Figures 2 and 3 present mean values of $24 \mathrm{~h}$, daytime and nighttime periods of BP monitoring before and after 8 weeks of RT in MetS and without MetS groups, respectively. Mean blood pressure (MBP) and DBP was decreased during nighttime in MetS group after the RT program (Figure 2). No statistically significant differences were observed in the group without MetS for $24 \mathrm{~h}$, daytime or nighttime analyses after the RT program (Figure 3).

Figure 4 shows $24 \mathrm{~h} \mathrm{BP}$ measurement for SBP, DBP and MBP before (pre-intervention) and after 8 weeks of RT in the MetS group. DBP values decreased at 11:00 pm (tendency from 75.5 to $67 ;-8.5 \mathrm{mmHg} p=0.06[-0.57,17.35-$

Table 1 Baseline characteristics of subjects

\begin{tabular}{|c|c|c|c|}
\hline & MetS & $\begin{array}{l}\text { Without } \\
\text { MetS }\end{array}$ & $P$ value \\
\hline & $(\mathbf{n}=\mathbf{9})$ & $(\mathbf{n}=\mathbf{8})$ & \\
\hline \multicolumn{4}{|l|}{ Anthropometric parameters } \\
\hline Age (years) & $37.0 \pm 8.7$ & $35.1 \pm 7.2$ & 0.98 \\
\hline Body Mass (kg) & $77.3 \pm 9.7^{*}$ & $58.2 \pm 6.2$ & 0.002 \\
\hline Height (m) & $1.60 \pm 0.08$ & $1.59 \pm 0.05$ & 0.51 \\
\hline Neck Circumference (cm) & $35.7 \pm 1.5^{*}$ & $33.1 \pm 1.7$ & 0.002 \\
\hline Waist Circumference (cm) & $90.9 \pm 9.2^{*}$ & $79.0 \pm 8.7$ & 0.009 \\
\hline Hip Circumference (cm) & $107.1 \pm 7.7^{*}$ & $98.0 \pm 6.3$ & 0.01 \\
\hline \multicolumn{4}{|c|}{ Body composition parameters } \\
\hline Body Mass Index $\left(\mathrm{kg} \cdot \mathrm{m}^{-2}\right)$ & $30.3 \pm 4.2^{*}$ & $24.2 \pm 2.5$ & 0.001 \\
\hline Body Fat (\%) & $37.1 \pm 3.1^{*}$ & $28.3 \pm 3.9$ & $<0.001$ \\
\hline Fat Body Mass (kg) & $28.9 \pm 5.6^{*}$ & $16.6 \pm 3.0$ & 0.02 \\
\hline Lean Body Mass (kg) & $48.7 \pm 6.0^{*}$ & $41.6 \pm 4.1$ & 0.02 \\
\hline \multicolumn{4}{|l|}{ Biochemical Parameters } \\
\hline Blood Glucose (mg ·dL) & $95.2 \pm 22.2$ & $85.6 \pm 4.5$ & 0.33 \\
\hline Triglycerides $(\mathrm{mg} \cdot \mathrm{dL}) \dagger$ & $115.0 \pm 37.4^{*}$ & $62.7 \pm 20.8$ & 0.006 \\
\hline $\mathrm{HDL}-\mathrm{C}(\mathrm{mg} \cdot \mathrm{dL})$ & $41.2 \pm 8.5^{*}$ & $59.0 \pm 12.3$ & 0.05 \\
\hline Z-Score MetS & $1.36 \pm 0.13^{*}$ & $1.16 \pm 0.06$ & 0.001 \\
\hline \multicolumn{4}{|l|}{ Hemodynamic variables } \\
\hline $\begin{array}{l}\text { Systolic Blood Pressure } \\
(\mathrm{mmHg})\end{array}$ & $131.8 \pm 16.1^{*}$ & $108.6 \pm 6.2$ & 0.002 \\
\hline $\begin{array}{l}\text { Diastolic Blood Pressure } \\
(\mathrm{mmHg})\end{array}$ & $84.1 \pm 11.3^{*}$ & $74.0 \pm 6.1$ & 0.03 \\
\hline $\begin{array}{l}\text { Mean Blood Pressure } \\
(\mathrm{mmHg})\end{array}$ & $100.1 \pm 11.9^{*}$ & $85.5 \pm 4.8$ & 0.005 \\
\hline Heart Rate (bpm) & $81.4 \pm 16.9$ & $76.0 \pm 14.3$ & 0.33 \\
\hline
\end{tabular}

The data are presented as mean \pm SD. *Significantly different between groups; ${ }^{\dagger}$ Values expressed as median; HDL high density lipoprotein, MetS metabolic syndrome.
$\mathrm{CI}]$ ), 02:00 am (from 70 to $61 ;-9 \mathrm{mmHg} ; \mathrm{p}=0.04[-13.45$, $0.04-\mathrm{CI}$ ), $07: 00$ am (from 71 to $65 ;-6 \mathrm{mmHg} ; \mathrm{p}=0.03$ $[0.71,12.51-\mathrm{Cl}]$ ) and 6:00 pm (from 81 to $71 ;-10 \mathrm{mmHg}$; $\mathrm{p}=0.02[-2.22,19.84-\mathrm{CI}])$ after eight weeks of training. MBP values decreased at 11:00 pm (from 91 to $81 ;-10$ mmHg; $\mathrm{p}=0.04[0.39,17.34-\mathrm{CI}]), 7: 00$ am (tendency from 85.2 to $80.2 ;-5.6 \mathrm{mmHg} ; \mathrm{p}=0.06$; [ $-12.02,0.57$ $\mathrm{CI}$ ), and $6 \mathrm{pm}$ (from 97.5 to $90.2 ;-7.3 \mathrm{mmHg} ; \mathrm{p}=0.03$; $[-13.87,-0.80-\mathrm{CI}])$ after the RT program. There was no statistically significant difference for SBP. No statistically significant alterations on MBP, DBP and SBP were observed after eight weeks of RT in the group without MetS (Figure 5).

\section{Discussion}

To the best of our knowledge, this is the first study to evaluate the effects of eight weeks of RT on $24 \mathrm{~h} \mathrm{BP}$ in individuals with MetS. Our initial hypothesis has been partially confirmed, since MBP and DBP decreased in the MetS group after the RT program, while SBP values remained stable. The decrease mainly occurred at 11:00 pm, 02:00 am, 07:00 am, and 6:00 pm. Furthermore, there was no effect of RT on $24 \mathrm{~h} \mathrm{BP}$ in women without MetS.

In daily practice, RT is employed to increase muscle strength parameters and improve metabolic profile parameters, which is favorable to the maintenance of functional capacity, prevention of sarcopenia, osteoporosis, obesity, hypertension and MetS [23-25]. However, contrary to endurance training, evidence for a BP-lowering effect of RT remains scarce and much less compelling. A meta-analysis from Cornelissen and Fagard [25] showed that RT reduces systolic and diastolic clinical BP by 3.2 and $3.5 \mathrm{mmHg}$, respectively in normotensive and hypertensive adults. Although relevant, these results should be analyzed with caution, because BP measurements were mainly collected at a specific period after training (most studies up to 60 minutes after training). Moreover, clinical relevance of BP decrease after a single or chronic RT should be maintained for many hours during day and nighttime periods $[13,15,26]$. The importance of chronic BP reductions and maintenance during the day can be estimated from large, prospective intervention studies investigating morbidity and mortality outcomes that suggest that small reductions in resting SBP and DBP of $3 \mathrm{mmHg}$ can reduce stroke by $8 \%$, coronary heart disease risk by $5 \%$, and all-cause mortality by $4 \%$ [26-29].

In the present study, women with MetS presented a night-time decrease of MBP ( -6 to $-10 \mathrm{mmHg}$ ) and DBP ( -5.6 to $-8.7 \mathrm{mmHg}$ ) mainly at 11:00 pm, 02:00 am (only diastolic), 07:00 am and 6:00 pm after eight weeks of RT. Most studies recommend $24 \mathrm{~h} \mathrm{BP}$ as a better predictor of morbidity and mortality than clinical BP [15,26,30]. However, there is still debate on the relative importance of daytime and nighttime 24 h BP. Furthermore, Fagard et al., [29] demonstrated that nighttime $\mathrm{BP}$ is in general a better 

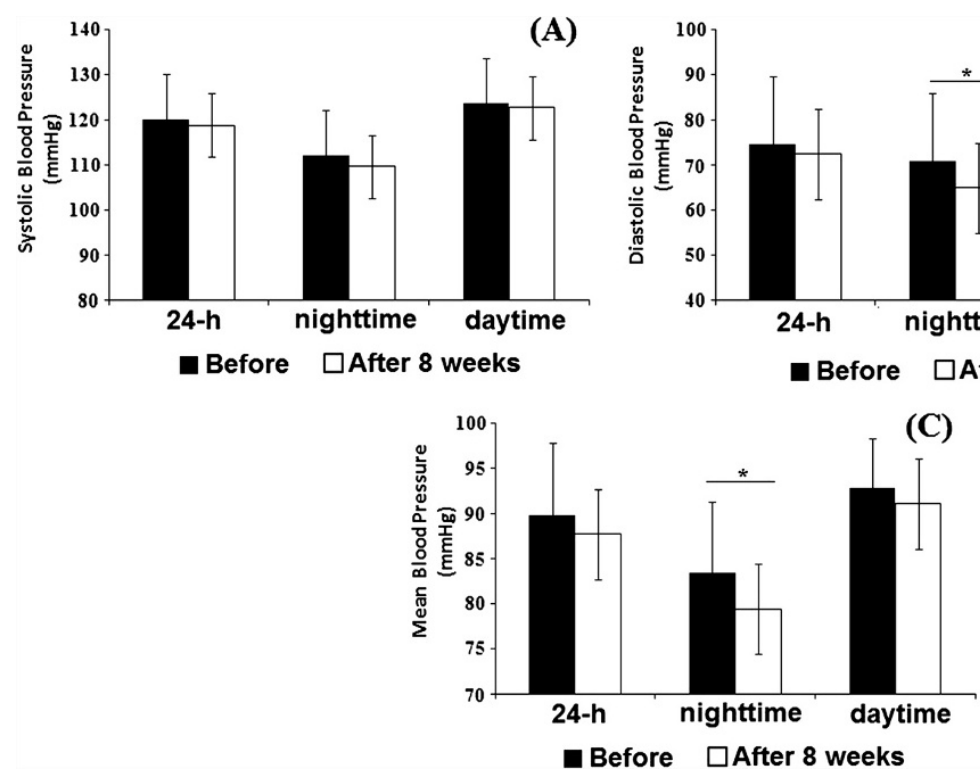

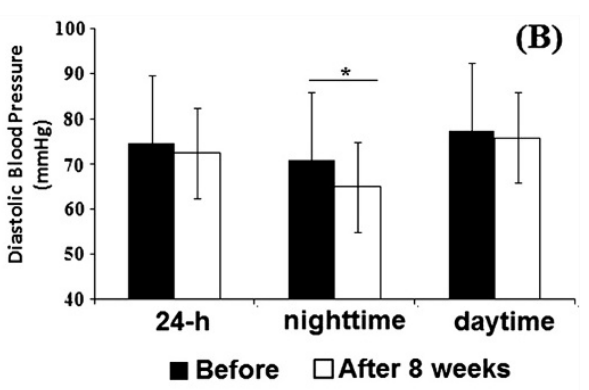

(C)

Figure 2 Twenty four hour (24 h), daytime and nighttime ambulatory blood pressure monitoring, before and after eight weeks of resistance training in individuals with metabolic syndrome. Systolic blood pressure (panel A); diastolic blood pressure (panel B); mean blood pressure (panel $\mathbf{C})$. ${ }^{*}$ different from pre-training $(p<0.05)$. Valeus are presented as mean $\pm S D$.

predictor of outcome than daytime pressure in hypertensive patients, and the night-day BP ratio predicts mortality, even after adjustment for $24 \mathrm{~h}$ BP [27,31]. The recent MAPEC (Ambulatory Blood Pressure Monitoring and Cardiovascular Events) study [32] revealed a significant relationship between decreasing BP during sleeping hours and reduced cardiovascular risk in subjects with normal or elevated BP, suggesting risk-reduction benefits even below the current diagnostic threshold of $120 \mathrm{mmHg}$. Thus, the decrease of BP during sleeping hours should be considered as a novel therapeutic target for reduction of cardiovascular risk [32].

In this sense, this reinforces the clinical relevance of the present results. The drop of BP during sleeping hours in women with MetS would be of great importance in preventing the development of hypertension and cardiovascular diseases [2,3]. Although the mechanisms associated
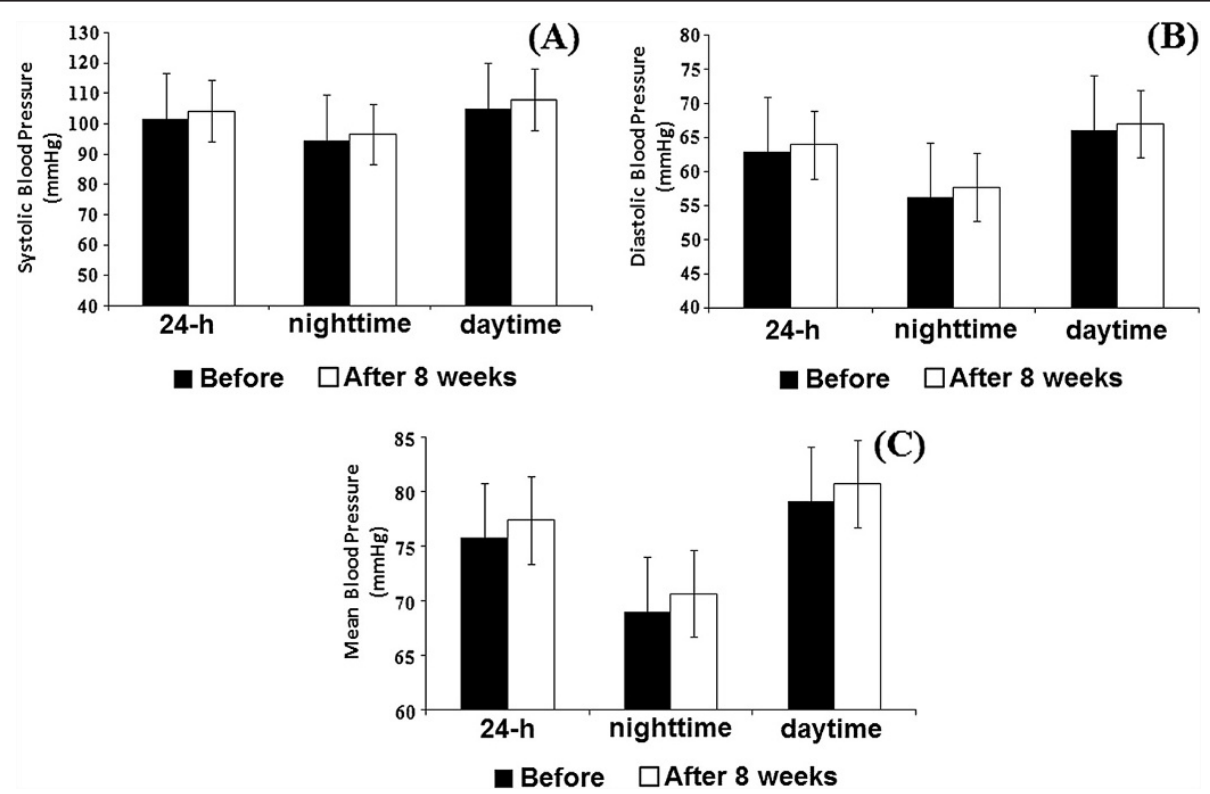

Figure 3 Twenty four hour (24 h), daytime and nighttime ambulatory blood pressure monitoring, before and after eight weeks of resistance training in individuals without metabolic syndrome. Systolic blood pressure (panel A); diastolic blood pressure (panel B); mean blood pressure (panel $\mathbf{C}$ ). Values are presented as mean \pm SD. 

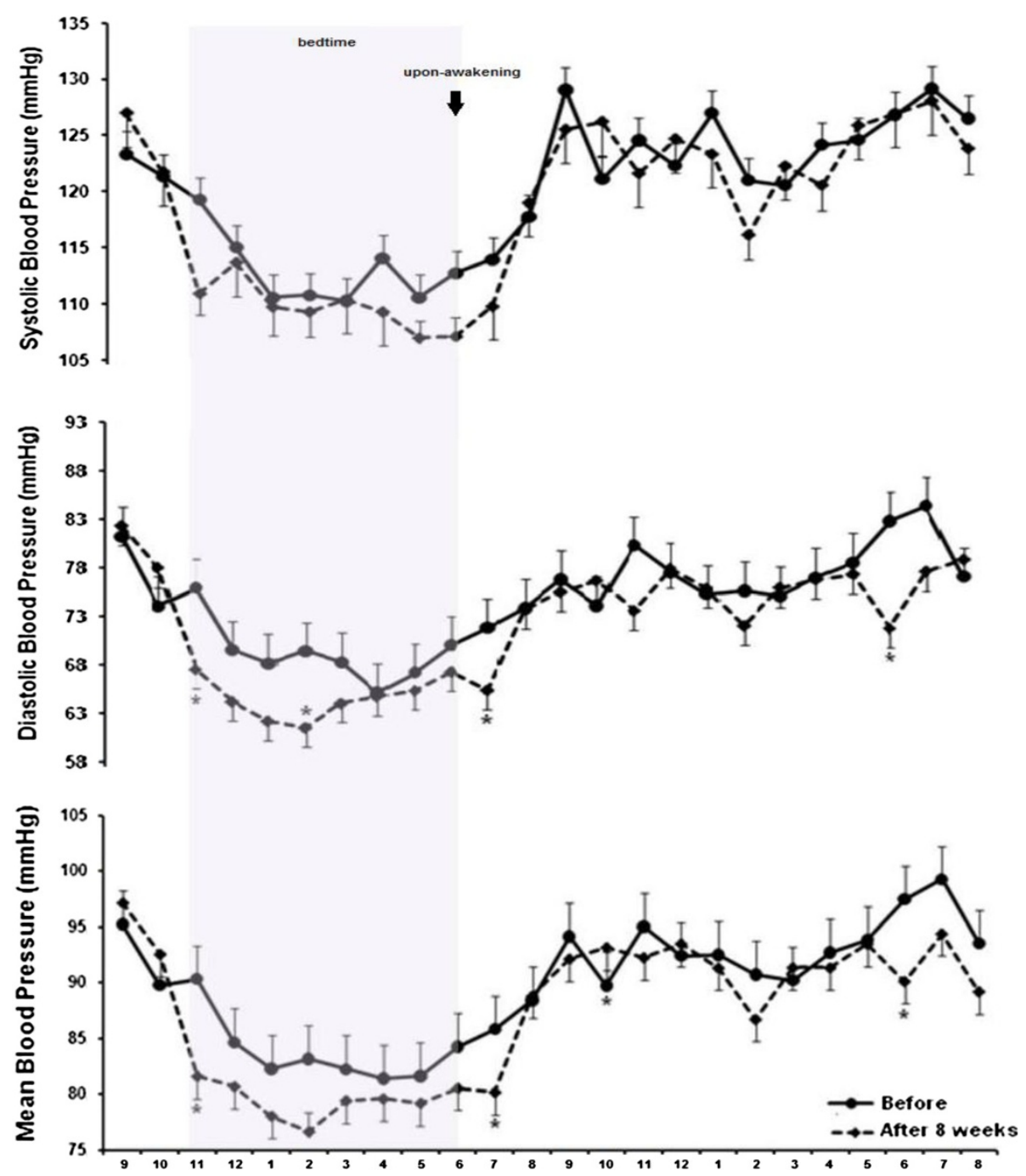

Figure 4 Twenty four hour blood pressure monitoring before and after eight weeks of resistance training in the metabolic syndrome group. *different from pre-training $(p \leq 0.05)$. Values are presented as mean $\pm S D$.

with post-exercise hypotension have not been the focus of the present study, it is possible to speculate that several hemodynamic and neuro-humoral factors, such as decreased sympathetic activity, plasma catecholamine levels and vascular tone interacted to produce this circadian variation in BP [30]. Additional proposals include a decreased cardiac output and peripheral vascular resistance [33-35], higher activity of the plasma kallikrein system mediating nitric oxide release [36], and alterations in cerebral blood flow induced by exercise [34]. More studies will be necessary to adequately address the mechanisms responsible for the reduction of MBP and DBP after RT.

Although acute hypotensive effects of aerobic [37] and resistance exercise $[38,39]$ has been widely shown in different populations, the chronic effects of RT on $24 \mathrm{~h} \mathrm{BP}$ are controversial and scarce. Pescatello et al., [40] investigated the acute effects of 30 minutes of aerobic exercise (cycle ergometer) at $40 \%$ or $60 \%$ of $\mathrm{VO}_{2}$ peak and found a nonsignificant drop of BP up to nine hours after exercise in men with MetS. Stensvold et al., [41] compared the effects of interval aerobic training, $\mathrm{RT}$ and aerobic $+\mathrm{RT}$ on risk factors of MetS and found a non-significant chronic decrease of BP in individuals with MetS. The difficulties in comparing the results from previously published studies with our results are that most studies with MetS and exercise were acute used limited time-course analyses of BP and different exercise protocols. To note, the RT used in the present study is practical and widely recommended, as we used one resistance exercise for each main muscle group, which reinforces the clinical relevance of the results.

The results of the present study showed no effect of RT on $24 \mathrm{~h} \mathrm{BP}$ during daytime and nighttime analyses in women without MetS. The failure to observe chronic hypotensive effects of RT is in accordance with previous 

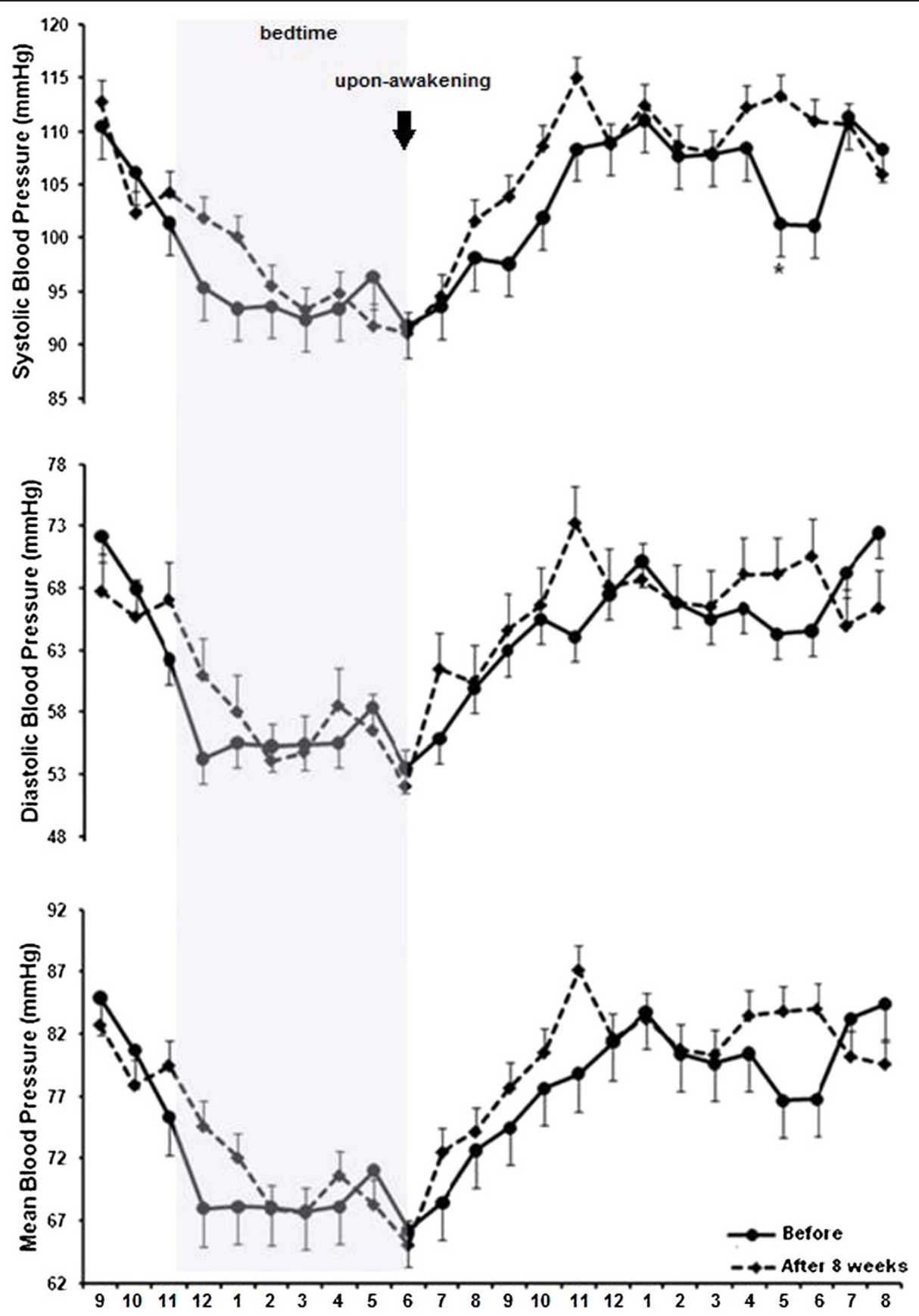

Figure 5 Twenty four hour blood pressure monitoring before and after eight weeks of resistance training in the group without metabolic syndrome. Values are presented as mean \pm SD.

studies investigating $24 \mathrm{~h} \mathrm{BP}$ in normotensive or mild hypertensive subjects $[16,42]$.

Some limitations of the present study should be considered. The circadian pattern of BP has a strong genetic dependency in terms of daytime, the amplitude of variation, and peak time during a $24 \mathrm{~h}$ period. No mechanisms regarding the chronic decrease of $\mathrm{BP}$ were investigated, the number of participants in each group was small and the intervention lasted only eight weeks.

Furthermore, the use of $48 \mathrm{~h} \mathrm{BP}$ monitoring instead of the more common $24 \mathrm{~h}$ could increase the reproducibility of the BP findings [42], and use of wrist actigraphy to precisely and individually determine the beginning and ending of the activity and sleep spans for each subject 
could enable the accurate calculation of the awake and sleeping BP means [26].

In conclusion, after 8 weeks of a resistance-training program, the mean blood pressure and diastolic blood pressure decreased during day and sleeping hours in women with metabolic syndrome. The preset results provide a possible non-pharmacological intervention strategy for decreasing sleep-time blood pressure that could be evaluated in patients by ambulatory monitoring. Future studies should be designed to reinforce our findings and evaluate the markers of cardiovascular morbidity and mortality. In addition, studies evaluating the effects of RT performed during different periods of the day (morning versus night) would improve the understanding regarding the circadian rhythm of blood pressure.

\section{Competing interests}

This research received no specific grant from any funding agency in the public, commercial, or not-for-profit sectors. The authors declare no competing interests.

\section{Authors' contributions}

RAT, GBP, JCdeS, CRC and JP were responsible for concept and design, statistical expertise, data analysis and interpretation and helped to write the manuscript. DV, VT: CSGC were significant manuscript reviewers/revisers and were responsible for data analysis and interpretation. All authors have read and approved the manuscript for publication.

\section{Acknowledgments}

We would like to acknowledge the support of Centro Universitário EuroAmericano (UNIEURO, Brasilia, Brazil) and CAPES/PROSUP. We are very grateful for the English revision by Dr Scott Lyons.

\section{Author details}

${ }^{1}$ Graduate Program on Physical Education and Health, Catholic University of Brasilia (UCB), Brasilia, Brazil. ${ }^{2}$ Federal University of Maranhão (UFM),

Maranhão, Brazil. ${ }^{3}$ School of Physical Education, State University of Campinas, Campinas, Brazil.

\section{Received: 7 March 2013 Accepted: 16 April 2013}

Published: 27 May 2013

\section{References}

1. Heidenreich PA, Trogdon JG, Khavjou OA, Butler J, Dracup K, Ezekowitz MD, Finkelstein EA, Hong Y, Johnston SC, Khera A, Lloyd-Jones DM, Nelson SA, Nichol G, Orenstein D, Wilson PW, Woo YJ: Forecasting the future of cardiovascular disease in the United States: a policy statement from the American Heart Association. Circulation 2011, 123:933-944.

2. Cornier MA, Dabelea D, Hernandez TL, Lindstrom RC, Steig AJ, Stob NR, Van Pelt RE, Wang H, Eckel RH: The metabolic syndrome. Endocr Rev 2008, 29:777-822.

3. Gami AS, Witt BJ, Howard DE, Erwin PJ, Gami LA, Somers VK, Montori VM: Metabolic syndrome and risk of incident cardiovascular events and death: a systematic review and meta-analysis of longitudinal studies. J Am Coll Cardiol 2007, 30:403-414.

4. Dutra ES, de Carvalho KM, Miyazaki E, Hamann EM, Ito MK: Metabolic syndrome in central Brazil: prevalence and correlates in the adult population. Diabetol Metab Syndr 2012, 4:20.

5. Strasser B, Siebert U, Schobersberger W: Resistance training in the treatment of the metabolic syndrome: a systematic review and metaanalysis of the effect of resistance training on metabolic clustering in patients with abnormal glucose metabolism. Sports Med 2010, 40:397-415.

6. Westheim A, Os I: Physical activity and the metabolic cardiovascular syndrome. J Cardiovasc Pharmacol 1992, 20(Suppl 8):S49-S53.
7. IVy $\mathrm{JL}$ : Role of exercise training in the prevention and treatment of insulin resistance and non-insulin-dependent diabetes mellitus. Sports Med 1997, 24:321-336.

8. Carroll S, Dudfield M: What is the relationship between exercise and metabolic abnormalities? A review of the metabolic syndrome. Sports Med 2004, 34:371-418.

9. Pattyn N, Cornelissen VA, Eshghi SR, Vanhees $L$ : The effect of exercise on the cardiovascular risk factors constituting the metabolic syndrome: a meta-analysis of controlled trials. Sports Med 2013, 43:121-133.

10. Tibana RA, Prestes J: Treinamento de Força e Síndrome Metabólica: uma revisão sistemática. Rev Bras Cardiol 2013, 26:66-76.

11. Smutok MA, Reece C, Kokkinos PF, Farmer C, Dawson P, Shulman R, DeVane-Bell J, Patterson J, Charabogos C, Goldberg AP, Hurley BF: Aerobic versus strength training for risk factor intervention in middle-aged men at high risk for coronary heart disease. Metabolism 1993, 42:177-184.

12. Katzel LI, Bleecker ER, Rogus EM, Goldberg AP: Sequential effects of aerobic exercise training and weight loss on risk factors for coronary disease in healthy, obese middle-aged and older men. Metabolism 1997 46:1441-1447.

13. Kenney MJ, Seals DR: Postexercise hypotension. Key features, mechanisms, and clinical significance. Hypertension 1993, 22:653-664.

14. Vilaplana JM: Blood pressure measurement. J Ren Care 2006, 32:210-213.

15. Waeber B, Burnier M: Ambulatory blood pressure monitoring to assess cardiovascular risk in women. Hypertension 2011, 57:377-378.

16. Cornelissen VA, Fagard $\mathrm{RH}$, Coeckelberghs $\mathrm{E}$, Vanhees L: Impact of resistance training on blood pressure and other cardiovascular risk factors: a meta-analysis of randomized, controlled trials. Hypertension 2011, 58:950-958.

17. Grundy SM, Cleeman JI, Daniels SR, Donato KA, Eckel RH, Franklin BA, Gordon DJ, Krauss RM, Savage PJ, Smith SC Jr, Spertus JA, Costa F, American Heart Association; National Heart, Lung, and Blood Institute: Diagnosis and management of the metabolic syndrome: an American Heart Association/National Heart, Lung, and Blood Institute Scientific Statement. Circulation 2005, 112:2735-2752.

18. Tibana RA, Teixeira TG, Farias DL, Silva AD, Madrid B, Vieira A, Franz CB, Balsamo S, Souza Júnior TP, Prestes J: Relation of neck circumference and relative muscle strength and cardiovascular risk factors in sedentary women. Einstein 2012, 10:329-334.

19. Pollock ML, Jackson AS: Research progress in validation of clinical methods of assessing body composition. Med Sci Sports Exerc 1984, 16:606-615.

20. Sociedade Brasileira de Cardiologia (SBC); Sociedade Brasileira de Hipertensão (SBH); Sociedade Brasileira de Nefrologia (SBN): V Guidelines for ambulatory blood pressure monitoring (ABPM) and III Guidelines for home blood pressure monitoring (HBPM). Arq Bras Cardiol 2011, 97:1-24.

21. American College of Sports Medicine: Progression models in resistance training for healthy adults. Med Sci Sports Exerc 2009, 41:687-701.

22. Tibana RA, Prestes J, Nascimento Dda C, Martins OV, De Santana FS, Balsamo S: Higher muscle performance in adolescents compared with adults after a resistance training session with different rest intervals. J Strength Cond Res 2012, 26:1027-1032.

23. Artero EG, Lee DC, Lavie CJ, España-Romero V, Sui X, Church TS, Blair SN: Effects of muscular strength on cardiovascular risk factors and prognosis. J Cardiopulm Rehabil Prev 2012, 32:351-358.

24. Tibana RA, Navalta J, Bottaro M, Vieira D, Tajra V, Silva AD, De Farias DL, Pereira GB, De Souza JC, Balsamo S, Cavaglieri CR, Prestes J: Effects of eight weeks of resistance training on the risk factors of metabolic syndrome in overweight/obese women - "A Pilot Study". Diabetol Metab Syndr 2013, 28:11.

25. Cornelissen VA, Fagard RH: Effect of resistance training on resting blood pressure: a meta-analysis of randomized controlled trials. J Hypertens 2005, 23:251-259.

26. Whelton PK, He J, Appel LJ, Cutler JA, Havas S, Kotchen TA, Roccella EJ, Stout R, Vallbona C, Winston MC, Karimbakas J, National High Blood Pressure Education Program Coordinating Committee: Primary prevention of hypertension: clinical and public health advisory from The National High Blood Pressure Education Program. JAMA 2002, 288:1882-1888.

27. Stamler R: Implications of the INTERSALT study. Hypertension 1991, 17(1 Suppl):116-|20.

28. Lewington S, Clarke R, Qizilbash N, Peto R, Prospective CR, Studies C: Agespecific relevance of usual blood pressure to vascular mortality: a meta- 
analysis of individual data for one million adults in 61 prospective studies. Lancet 2002, 360:1903-1913.

29. Fagard RH, Celis H, Thijs L, Staessen JA, Clement DL, De Buyzere ML, De Bacquer DA: Daytime and nighttime blood pressure as predictors of death and cause-specific cardiovascular events in hypertension. Hypertension 2008, 51:55-61.

30. Giles T: Relevance of blood pressure variation in the circadian onset of cardiovascular events. J Hypertens Suppl 2005, 23:S35-S39.

31. Ribeiro F, Campbell CS, Mendes G, Arsa G, Moreira SR, Da Silva FM, Prestes J, Da Costa SR, Simões HG: Exercise lowers blood pressure in university professors during subsequent teaching and sleeping hours. Int I Gen Med 2011, 4:711-716.

32. Hermida RC, Ayala DE, Mojón A, Fernández JR: Decreasing sleep-time blood pressure determined by ambulatory monitoring reduces cardiovascular risk. J Am Coll Cardiol 2011, 58:1165-1173.

33. Brum PC, Da Silva GJ, Moreira ED, Ida F, Negrão CE, Krieger EM: Exercise training increases baroreceptor gain sensitivity in normal and hypertensive rats. Hypertension 2000, 36:1018-1022.

34. Chen CY, Bonham AC: Postexercise hypotension: central mechanisms. Exerc Sport Sci Rev 2010, 38:122-127.

35. Halliwill JR: Mechanisms and clinical implications of post-exercise hypotension in humans. Exerc Sport Sci Rev 2001, 29:65-70.

36. Halliwill JR, Minson CT, Joyner MJ: Eff ect of systemic nitric oxide ynthase inhibition on postexercise hypotension in humans. J Appl Physiol 2000, 89:1830-1836

37. Rodriguez D, Silva V, Prestes J, Rica RL, Serra AJ, Bocalini DS, Pontes FL Jr: Hypotensive response after water-walking and land walking exercisesessions in healthy trained and untrained women. Int I Gen Med 2011, 4:549-554

38. Tibana RA, Pereira GB, Bottaro M, Navalta J, Prestes J: Acute effects of resistance exercise on clinical and 24-hour ambulatory blood pressure in middle-aged overweight and obese women. Int J Sports Med 2013, 34:460-4.

39. Tibana RA, Boullosa DA, Leicht AS, Prestes J: Women with metabolic syndrome present different autonomic modulation and blood pressure response to an acute resistance exercise session. Clin Physiol Funct Imaging. in press.

40. Pescatello LS, Blanchard BE, Van Heest JL, Maresh CM, Gordish-Dressman H, Thompson PD: The metabolic syndrome and the immediate antihypertensive effects of aerobic exercise: a randomized control design. BMC Cardiovasc Disord 2008, 8:12.

41. Stensvold D, Tjønna AE, Skaug EA, Aspenes S, Stølen T, Wisløff U, Slørdahl $S A$ : Strength training versus aerobic interval training to modify risk factors of metabolic syndrome. J Appl Physio/ 2010, 108:804-810.

42. Hermida RC, Calvo C, Ayala DE, Fernández JR, Ruilope LM, López JE: Evaluation of the extent and duration of the "ABPM effect" in hypertensive patients. J Am Coll Cardiol 2002, 40:710-717.

doi:10.1186/1758-5996-5-27

Cite this article as: Tibana et al: Resistance training decreases 24-hour blood pressure in women with metabolic syndrome. Diabetology \& Metabolic Syndrome 2013 5:27.

\section{Submit your next manuscript to BioMed Central and take full advantage of:}

- Convenient online submission

- Thorough peer review

- No space constraints or color figure charges

- Immediate publication on acceptance

- Inclusion in PubMed, CAS, Scopus and Google Scholar

- Research which is freely available for redistribution

Submit your manuscript at www.biomedcentral.com/submit
C Biomed Central 